\title{
Weaker assumptions for convergence of extended block Kaczmarz and Jacobi projection algorithms
}

\author{
Doina Carp, Ioana Pomparău, Constantin Popa
}

\begin{abstract}
Recent developments in the field of image reconstruction have given rise to the use of projective iterative methods, such as Kaczmarz and Jacobi, when solving inconsistent linear least squares problems. In this paper we try to generalize previous results concerning extended block versions of these two algorithms. We replace the inverse operator with the Moore-Penrose pseudoinverse and try to prove convergence under weaker assumptions. In order to accomplish this task, we show that these algorithms are special cases of a general iterative process for which convergence is already established.
\end{abstract}

\section{Introduction}

Many important real-world problems gives after appropriate discretization techniques large, sparse and ill-conditioned systems of linear equations

$$
A x=b,
$$

where $A$ is an $m \times n$ real matrix and $b \in \mathbb{R}^{m}$. But, because of both discretization errors and measurements, the right hand side $b$ can be affected by noise and the system (1) becomes inconsistent. For this it must be reformulated and

Key Words: Inconsistent linear least squares problem; extended block Kaczmarz algorithm; extended block Jacobi with relaxation parameters algorithm; Moore-Penrose pseudoinverse; clustering.

2010 Mathematics Subject Classification: 65F10, 65F20.

Received: 15.11 .2015

Accepted: 30.04.2016 
a new (more general) type of solution defined. In order to present these constructions we will introduce the following notations which will be used on the whole paper. Therefore $A^{T}, A_{i}, A^{j}, \mathcal{R}(A), \mathcal{N}(A), P_{V}$ will denote the transpose, $i$-th row, $j$-th column, range and null space of $A$, the projection onto a vector subspace $V$; also $\langle\cdot, \cdot\rangle$ and $\|\cdot\|$ will denote the Euclidean scalar product and norm and all the vectors appearing in the paper will be considered as column vectors. We will assume in the rest of the paper that

$$
A_{i} \neq 0, A^{j} \neq 0 \text {, for all } i \in\{1,2, \ldots m\} \text {, and } j \in\{1,2, \ldots n\} \text {. }
$$

The inconsistency of the system (1) means that $b \notin \mathcal{R}(A)$, i.e.

$$
P_{\mathcal{N}\left(A^{T}\right)}(b)=b-P_{\mathcal{R}(A)}(b) \neq 0,
$$

thus

$$
\|A x-b\|^{2}=\left\|A x-P_{\mathcal{R}(A)}(b)\right\|^{2}+\left\|P_{\mathcal{N}\left(A^{T}\right)}(b)\right\|^{2}>0, \forall x \in \mathbb{R}^{n} .
$$

Thus, instead of $A x=b \Leftrightarrow\|A x-b\|=0$ we will reformulate the system (1) in the form: find $x \in \mathbb{R}^{n}$ such that

$$
\|A x-b\|=\min \left\{\|A z-b\|, z \in \mathbb{R}^{n}\right\},
$$

i.e. as a linear least squares problem. From (4) it results that the problem (5) is equivalent with the system

$$
A x=P_{\mathcal{R}(A)}(b),
$$

which tells us that it has always solutions. These more general solutions will be called least squares solutions and their set denoted by $\operatorname{LS} S(A ; b)$. Moreover, it can be proved that it exists a unique element $x_{L S} \in L S S(A ; b)$ with the properties that it is orthogonal on $\mathcal{N}\left(A^{T}\right)$ and has minimal Euclidean norm among the other solutions. It is called the minimal norm solution.

In the present paper we are concerned with the approximation of solutions of the problem (5), using block-type extended Kaczmarz and Jacobi projection methods.

Block Kaczmarz and Jacobi projection methods for solving inconsistent linear least squares problems as (5) have been first considered in [4] and extended for weighted formulation of (5) in [3]. But, both of these papers analyse block versions of original Kaczmarz or Jacobi projection methods. Extended block versions of these two algorithms were introduced in [6] and convergence was proved under nonsingularity assumptions on matrices resulted from row and column block decompositions. These assumptions are rather restrictive in practical applications, and the purpose of this paper is to eliminate them from 
the algorithms. In this respect, we will use the results from the paper [5], in which the authors proposed a general iterative procedure for finding a numerical solution of (5), which includes the algorithms of Kaczmarz, Cimmino, Jacobi Projective and Diagonal Weighting (see, e.g., [9], [5], [4] and [7], respectively). Moreover, they propose and theoretically analyse an extended version of this method, which generates a sequence of approximations convergent to an element from $\operatorname{LSS}(A ; b)$.

In this paper we show that, after replacing the inverse operator with the Moore-Penrose pseudoinverse, the earlier results on this topic, see [6, Theorem 3.4 and Theorem 6.7], still hold without the nonsingularity hypotheses. This is accomplished by firstly proving that the two algorithms are special cases of the extended iterative method from [5] above mentioned.

The paper is organized as follows: in Section 2 we briefly present Extended Block Kaczmarz (EBK) and Extended Block Jacobi with Relaxation Parameters (EBJRP) algorithms illustrated in [6] and the Extended General (EGEN) procedure from [5]; in Section 3 we show that the EBK and EBJRP solvers, defined with the Moore-Penrose pseudoinverses, belong to the family of EGEN methods.

\section{Solving the inconsistent linear least squares problem}

We consider block row decompositions of the matrix $A$ and corresponding vector $b$. In this respect, let $p \geq 2,1 \leq m_{i} \leq m$, with $i \in\{1,2, \ldots, p\}$, such that $m_{1}+m_{2}+\cdots+m_{p}=m$,

$$
A=\left[\begin{array}{c}
A_{1} \\
A_{2} \\
\vdots \\
A_{p}
\end{array}\right], \text { and } b=\left[\begin{array}{c}
b_{1} \\
b_{2} \\
\vdots \\
b_{p}
\end{array}\right]
$$

where $A_{i}$ are $m_{i} \times n$ matrices and $b_{i} \in \mathbb{R}^{m_{i}}$.

Similarly, for $q \geq 2$ and $n=n_{1}+n_{2}+\cdots+n_{q}$, with $1 \leq n_{j}<n$ for any $j \in\{1,2, \ldots, q\}$, the block column decomposition of $A$ is given by

$$
A^{T}=\left[\begin{array}{c}
B_{1} \\
B_{2} \\
\vdots \\
B_{q}
\end{array}\right]
$$

where $B_{j}$ are $n_{j} \times m$ real matrices. 
In [6] the author introduced extended block versions of the Kaczmarz and Jacobi with relaxation parameters algorithms. They proved that under the hypotheses

$$
\operatorname{det}\left(A_{i} A_{i}^{T}\right) \neq 0, \forall i \in\{1,2, \ldots, p\}
$$

and

$$
\operatorname{det}\left(B_{j} B_{j}^{T}\right) \neq 0, \forall j \in\{1,2, \ldots, q\}
$$

these methods converge to an element of the linear least square solutions set of the problem (5).

Let the linear applications $f_{0}^{i}(b ; \cdot), F_{0}(b ; \cdot): \mathbb{R}^{n} \rightarrow \mathbb{R}^{n}$ be defined by

$$
\begin{gathered}
f_{0}^{i}(b ; x)=x+A_{i}^{T}\left(A_{i} A_{i}^{T}\right)^{-1}\left(b_{i}-A_{i} x\right), \forall i \in\{1,2, \ldots, p\}, \\
F_{0}(b ; x)=\left(f_{0}^{1} \circ f_{0}^{2} \circ \cdots \circ f_{0}^{p}\right)(b ; x)
\end{gathered}
$$

and the linear mapping

$$
\Phi_{0}=\prod_{j=1}^{q} I-B_{j}^{T}\left(B_{j} B_{j}^{T}\right)^{-1} B_{j} .
$$

The extended block version of the Kaczmarz Algorithm defined according to [6] now follows.

Extended Block Kaczmarz Algorithm(EBK)

Initialization: $x^{0} \in R^{n}$ is arbitrary and $y^{0}=b$.

Iterative step For every $k \geq 0$,

$$
\begin{aligned}
y^{k+1} & =\Phi_{0} y^{k}, \\
b^{k+1} & =b-y^{k+1}, \\
x^{k+1} & =F_{0}\left(b^{k+1} ; x^{k}\right) .
\end{aligned}
$$

Now, for the real parameters $\omega, \alpha \neq 0$, consider

$$
\begin{gathered}
Q_{0}^{\omega}=I-\omega \sum_{i=1}^{p} A_{i}^{T}\left(A_{i} A_{i}^{T}\right)^{-1} A_{i} \\
R_{0}^{\omega}=\omega \operatorname{col}\left[A_{1}^{T}\left(A_{1} A_{1}^{T}\right)^{-1}\left|A_{2}^{T}\left(A_{2} A_{2}^{T}\right)^{-1}\right| \ldots \mid A_{p}^{T}\left(A_{p} A_{p}^{T}\right)^{-1}\right]
\end{gathered}
$$

and

$$
\Phi_{0}^{\alpha}=I-\alpha \sum_{j=1}^{q} B_{j}^{T}\left(B_{j} B_{j}^{T}\right)^{-1} B_{j} .
$$


An extended version of the Jacobi method with relaxation parameters was given in [6].

\section{Extended Block Jacobi Algorithm with Relaxation Parameters}

(EBJRP)

Initialization: $x^{0} \in R^{n}$ is arbitrary and $y^{0}=b$.

Iterative step For every $k \geq 0$,

$$
\begin{aligned}
y^{k+1} & =\Phi_{0}^{\alpha} y^{k}, \\
b^{k+1} & =b-y^{k+1}, \\
x^{k+1} & =Q_{0}^{\omega} x^{k}+R_{0}^{\omega} b^{k+1} .
\end{aligned}
$$

Unfortunately, in real examples, conditions of the type (9) - (10) are usually not true or hard to verify. Following the considerations from [6], we will show that if we use the Moore-Penrose pseudoinverse rather than the inverse operator, the convergence results remain true without the assumptions (9) (10). This is accomplished by proving that the two algorithms are particular cases of a general projection method, described below.

For the matrices $Q, R$ and $U$ of dimensions $n \times n, n \times m$ and $m \times m$, respectively, the authors defined in [5] the following extended general iterative method.

Extended General Algorithm (EGEN)

Initialization: $x^{0} \in R^{n}$ is arbitrary and $y^{0}=b$.

Iterative step For every $k \geq 0$,

$$
\begin{aligned}
y^{k+1} & =U y^{k}, \\
b^{k+1} & =b-y^{k+1}, \\
x^{k+1} & =Q x^{k}+R b^{k+1} .
\end{aligned}
$$

Let us suppose that $Q, R$ and $U$ satisfy the following general assumptions

$$
\begin{gathered}
Q+R A=I, \\
\forall y \in \mathbb{R}^{m}, R y \in \mathcal{R}\left(A^{T}\right), \\
\text { if } \tilde{Q}=Q P_{\mathcal{R}\left(A^{T}\right)} \text { then }\|\tilde{Q}\|<1, \\
\text { if } x \in \mathcal{N}\left(A^{T}\right) \text { then } U x=x, \\
\text { if } x \in \mathcal{R}(A) \text { then } U x \in \mathcal{R}(A),
\end{gathered}
$$




$$
\text { if } \tilde{U}=U P_{\mathcal{R}(A)} \text { then }\|\tilde{U}\|<1
$$

where by $\|T\|=\sup _{x \neq 0} \frac{\|T x\|}{\|x\|}$ we denoted the spectral norm of a matrix $T$. The authors proved in [5] the next convergence result for the EGEN algorithm.

Theorem 1. [5, Theorem 2.6] Let us suppose that the matrices $Q$ and $R$ satisfy equations (26) - (28) and for $U$ the properties (29) - (31) hold. Then, for any $x^{0} \in \mathbb{R}^{n}$, the sequence $\left(x^{k}\right)_{k \geq 0}$ generated with the algorithm EGEN converges and

$$
\lim _{k \rightarrow \infty} x^{k}=P_{\mathcal{N}(A)}\left(x^{0}\right)+x_{L S}
$$

It is known (for details and proofs see, e.g., [8]) that $L S S(A ; b)=\mathcal{N}(A)+$ $x_{L S}$, therefore, the EGEN procedure is a solver for the inconsistent problem (5).

\section{Main results}

The next result provides us with important information about the properties of the Moore-Penrose pseudoinverse (for details and proofs see e.g. [1, 2]).

Lemma 1. (i) The following relations hold for the Moore-Penrose pseudoinverse of an $m \times n$ real matrix $A$, denoted by $A^{\dagger}$.

$$
A^{\dagger}=A^{T}\left(A A^{T}\right)^{\dagger}, A^{T}=A^{\dagger} A A^{T}, \text { and } A^{T}=A^{T} A A^{\dagger}
$$

(ii) The orthogonal projectors $P_{\mathcal{R}\left(A^{T}\right)}$ and $P_{\mathcal{N}(A)}$ are given by

$$
P_{\mathcal{R}\left(A^{T}\right)}=A^{\dagger} A, P_{\mathcal{N}(A)}=I-A^{\dagger} A \text {. }
$$

In the rest of the paper we will denote by $f^{i}, F, \Phi, Q^{\omega}, R^{\omega}$ and $\Phi^{\alpha}$, the linear operators $f_{0}^{i}, F_{0}, \Phi_{0}, Q_{0}^{\omega}, R_{0}^{\omega}$ and $\Phi_{0}^{\alpha}$ defined according to (11) - (13) and $(17)-(19)$, respectively, in which we replaced the inverse mapping with the Moore-Penrose pseudoinverse. For any $m \times n$ matrix $M$, let $M^{\dagger}$ be its (unique) $n \times m$ pseudoinverse.

For every $i \in\{1,2, \ldots, p\}$ and $j \in\{1,2, \ldots, q\}$, we define the matrices

$$
\begin{gathered}
P_{i}=I-A_{i}^{T}\left(A_{i} A_{i}^{T}\right)^{\dagger} A_{i}, \phi_{j}=I-B_{j}^{T}\left(B_{j} B_{j}^{T}\right)^{\dagger} B_{j}, \\
\bar{P}_{i}=A_{i}^{T}\left(A_{i} A_{i}^{T}\right)^{\dagger} A_{i}, \bar{\phi}_{j}=B_{j}^{T}\left(B_{j} B_{j}^{T}\right)^{\dagger} B_{j}
\end{gathered}
$$

and make the following remarks. Equations (35), (33) and (34) yield that $P_{i}$ is the orthogonal projector on $\mathcal{N}\left(A_{i}\right)$. From (35), (33) and (34), it results that $\phi_{j}$ is the orthogonal projector on $\mathcal{N}\left(B_{j}\right)$. Similarly, (36), (33) and (34) 
give that $\bar{P}_{i}$ is the orthogonal projector on $\mathcal{R}\left(A_{i}^{T}\right)$. Using (36), (33) and (34), we obtain that $\bar{\phi}_{j}$ is the orthogonal projector on $\mathcal{R}\left(B_{j}^{T}\right)$. Also, the following properties hold

$$
\begin{aligned}
& P_{i}^{2}=P_{i}, P_{i}^{T}=P_{i} \text { and }\left\|P_{i}\right\|=1 ; \phi_{j}^{2}=\phi_{j}, \phi_{j}^{T}=\phi_{j} \text { and }\left\|\phi_{j}\right\|=1 ; \\
& \bar{P}_{i}^{2}=\bar{P}_{i}, \bar{P}_{i}^{T}=\bar{P}_{i} \text { and }\left\|\bar{P}_{i}\right\|=1 ; \bar{\phi}_{j}^{2}=\bar{\phi}_{j}, \bar{\phi}_{j}^{T}=\bar{\phi}_{j} \text { and }\left\|\bar{\phi}_{j}\right\|=1
\end{aligned}
$$

We will now consider the corresponding linear mappings

$$
\begin{gathered}
\Delta_{i}=A_{i}^{T}\left(A_{i} A_{i}^{T}\right)^{\dagger}, Q_{i}=P_{1} P_{2} \ldots P_{i}, \forall i \in\{1,2, \ldots, p\}, \\
Q=P_{1} P_{2} \ldots P_{p}, \\
R=\operatorname{col}\left[\Delta_{1}\left|Q_{1} \Delta_{2}\right| \ldots \mid Q_{p-1} \Delta_{p}\right], \\
\Phi_{j}=\phi_{1} \phi_{2} \ldots \phi_{j}, \forall j \in\{1,2, \ldots, q\} \\
\tilde{Q}^{\omega}=Q^{\omega} P_{\mathcal{R}}\left(A^{T}\right) \text { and } \tilde{\Phi}^{\alpha}=\Phi^{\alpha} P_{\mathcal{R}}(A) .
\end{gathered}
$$

Consequently, from (13), (37), (17), (38), (18), (39), (19) we have

$$
\begin{gathered}
\Phi=\phi_{1} \phi_{2} \ldots \phi_{q}, \\
Q^{\omega}=I-\omega \sum_{i=1}^{p} \bar{P}_{i}, \\
R^{\omega}=\omega \operatorname{col}\left[\Delta_{1}\left|\Delta_{2}\right| \ldots \mid \Delta_{p}\right]
\end{gathered}
$$

and

$$
\Phi^{\alpha}=I-\alpha \sum_{j=1}^{q} \bar{\phi}_{j} .
$$

We will require the next three results to prove that the extended block versions of the Kaczmarz and Jacobi algorithms are special cases of the EGEN method.

Lemma 2. If the $n \times n$ matrix $Q$ and the $n \times m$ matrix $R$ satisfy (26) - (27), the following properties are true.

$$
\text { if } x \in \mathcal{N}(A) \text { then } Q x=x
$$

and

$$
\text { if } x \in \mathcal{R}\left(A^{T}\right) \text { then } Q x \in \mathcal{R}\left(A^{T}\right) \text {. }
$$


Lemma 3. The following equality holds

$$
\mathcal{N}(A)=\bigcap_{i=1}^{p}\left\{x \in \mathbb{R}^{n}, P_{i} x=x\right\}
$$

Proof. If $x \in \mathcal{N}(A)$, then, for any $i \in\{1,2, \ldots, p\}, x \in \mathcal{N}\left(A_{i}\right)$ and from (35) we have that $P_{i} x=x$.

Let us now take $x \in \mathbb{R}^{n}$ such that $P_{i} x=x$ for every $i \in\{1,2, \ldots, p\}$. We then have $x-A_{i}^{T}\left(A_{i} A_{i}^{T}\right)^{\dagger} A_{i} x=x$, which together with (33) and (34) yields $P_{\mathcal{R}\left(A^{T}\right)}(x)=0$. Hence, $x \in \mathcal{N}\left(A_{i}\right)$ for every $x \in\{1,2, \ldots, p\}$ and the proof is complete.

Lemma 4. [9, Lemma 2, Corollary 3] If $P_{1}, P_{2}, \ldots P_{p}$ are the orthogonal projectors defined according to (35) and $Q$ is the linear mapping from (40), then

$$
\|Q x\|=\|x\| \text { if and only if } x \in \mathcal{N}(A)
$$

and

$$
\|Q\| \leq 1
$$

The following theorem ensures the convergence of the extended block Kaczmarz algorithm defined using the Moore-Penrose pseudoinverse, without the assumptions (9) - (10).

Theorem 2. If $Q, R$ and $\Phi$ are linear applications defined according to (40), (41) and (44), respectively, then

(i) we have the equality

$$
F(b ; x)=Q x+R b
$$

(ii) $Q$ and $R$ satisfy (26) - (28),

(iii) for the matrix $\Phi$ the properties (29) - (31) hold.

Proof. (i) For any $i \in\{1,2, \ldots, n\},(35)$ and (39) yield

$$
f_{i}(b ; x)=\left(I-A_{i}^{T}\left(A_{i} A_{i}^{T}\right)^{\dagger} A_{i}\right) x+A_{i}^{T}\left(A_{i} A_{i}^{T}\right)^{\dagger} b_{i}=P_{i} x+\Delta_{i} b_{i} .
$$

Hence,

$$
f_{1} \circ f_{2}=P_{1}\left(P_{2} x+\Delta_{2} b_{2}\right)+\Delta_{1} b_{1}=Q_{2} x+Q_{1} \Delta_{2} b_{2}+\Delta_{1} b_{1} .
$$

Using a recursive argument we obtain from (41) that

$$
f_{1} \circ f_{2} \circ \cdots \circ f_{p}=Q x+\sum_{i=1}^{p} Q_{i-1} \Delta_{i} b_{i}=Q x+R b,
$$


where $Q_{0}=I$, proving the equality (53).

(ii) In order to prove the assumption (26), from (41), (40), (35) and (39) we will write

$$
\begin{aligned}
R A & =\sum_{i=1}^{p} Q_{i-1} \Delta_{i} A_{i}=\sum_{i=1}^{p} P_{1} P_{2} \ldots P_{i-1} A_{i}^{T}\left(A_{i} A_{i}^{T}\right)^{\dagger} A_{i} \\
& =\sum_{i=1}^{p} P_{1} P_{2} \ldots P_{i-1}\left(I-P_{i}\right)=I-Q .
\end{aligned}
$$

Using (41) it may be easily proved that

$$
\mathcal{R}(R)=\sum_{i=1}^{p} \mathcal{R}\left(Q_{i-1} \Delta_{i}\right)
$$

If $y \in \mathcal{R}\left(Q_{i-1} \Delta_{i}\right)$, where $i \in\{1,2, \ldots, p\}$, there exists $z \in \mathbb{R}^{m}$ such that $Q_{i-1} \Delta_{i} z=y$. Since $P_{1}, P_{2}, \ldots, P_{i-1}$ are orthogonal projectors, for any $x \in$ $\mathcal{N}(A)$, the relations (40), (35), (39) and (50) give us

$$
\begin{aligned}
\langle y, x\rangle & =\left\langle Q_{i-1} \Delta_{i} z, x\right\rangle=\left\langle\Delta_{i} z, P_{i-1}^{T} \ldots P_{1}^{T} x\right\rangle \\
& =\left\langle A_{i}^{T}\left(A_{i} A_{i}^{T}\right)^{\dagger} z, x\right\rangle=\left\langle\left(A_{i} A_{i}^{T}\right)^{\dagger} z, A_{i} x\right\rangle=0 .
\end{aligned}
$$

Since $x \in \mathcal{N}(A)$ was arbitrarily chosen, we get $y \in \mathcal{R}\left(A^{T}\right)$, which, together with (58), implies that $\mathcal{R}(R) \subset \mathcal{R}\left(A^{T}\right)$.

Using the definition $\tilde{Q}=Q P_{\mathcal{R}\left(A^{T}\right)}$ and (52) we obtain

$$
\begin{aligned}
\|\tilde{Q}\| & =\sup \left\{\|\tilde{Q} x\|, \text { for all } x \in \mathbb{R}^{n} \text { with }\|x\|=1\right\} \\
& =\sup \left\{\left\|\tilde{Q} P_{\mathcal{R}\left(A^{T}\right)}(x)+\tilde{Q} P_{\mathcal{N}(A)}(x)\right\|, \text { for all } x \in \mathbb{R}^{n} \text { with }\|x\|=1\right\} \\
& =\sup \left\{\left\|\tilde{Q} P_{\mathcal{R}\left(A^{T}\right)}(x)\right\|, \text { for all } x \in \mathbb{R}^{n} \text { with }\left\|P_{\mathcal{R}\left(A^{T}\right)}(x)\right\| \leq 1\right\} \\
& =\sup \left\{\left\|Q P_{\mathcal{R}\left(A^{T}\right)}(x)\right\|, \text { for all } x \in \mathbb{R}^{n} \text { with }\left\|P_{\mathcal{R}\left(A^{T}\right)}(x)\right\|=1\right\} \\
& \leq 1 .
\end{aligned}
$$

Let us suppose that $\|\tilde{Q}\|=1$. From the above inequality it exists $x \in \mathcal{R}\left(A^{T}\right)$ such that $\|Q x\|=\|x\|$. Consequently, (51) yields $x \in \mathcal{N}(A)$, which implies that $x=0$, in contradiction with the assumption that $\|x\|=1$. Therefore, $\|\tilde{Q}\|<1$

(iii) We will firstly observe that, given the linear system of equations $A^{T} y=0$ and the block column decomposition (8) of $A$, the construction 
of the matrix $\Phi$ is equivalent to that of $Q$ for the system $A x=b$. Likewise, the matrix $S=\operatorname{col}\left[B_{1}^{T}\left(B_{1} B_{1}^{T}\right)^{\dagger}\left|\Phi_{1} B_{2}^{T}\left(B_{2} B_{2}^{T}\right)^{\dagger}\right| \ldots \mid \Phi_{q-1} B_{q}^{T}\left(B_{q} B_{q}^{T}\right)^{\dagger}\right]$ corresponds to $R$. Hence, from (ii) and Lemma 2, the matrix $\Phi$ satisfies (29) - (31) with $A^{T}$ instead of $A$ and the proof is complete.

In the case of the EBJRP algorithm we will confirm similar statements. The assumptions (28) and (31) will be proved using results from $[4,6]$.

Theorem 3. The following properties are true

(i) $Q^{\omega}$ and $R^{\omega}$ satisfy (26) - (27),

(ii) for the matrix $\Phi^{\alpha}$ the assumptions (29) - (30) hold.

Proof. (i) Using (46), (39), (36) and (45) we obtain

$$
R^{\omega} A=\omega \sum_{i=1}^{p} \Delta_{i} A_{i}=\omega \sum_{i=1}^{p} \bar{P}_{i}=I-Q^{\omega} .
$$

The definition of (39) yields $\mathcal{R}\left(R^{\omega}\right)=\sum_{i=1}^{p} \mathcal{R}\left(\Delta_{i}\right)$. Now, for any $i \in$ $\{1,2, \ldots, p\}$, let $y \in \mathcal{R}\left(\Delta_{i}\right)$ and $x \in \mathcal{N}(A)$. It results that there exists $z \in \mathbb{R}^{m}$ such that $\Delta_{i} z=y$ and from (39) we have $\langle y, x\rangle=\left\langle\Delta_{i} z, x\right\rangle=$ $\left\langle\left(A_{i} A_{i}^{T}\right)^{\dagger} z, A_{i} x\right\rangle=0$. Hence, $y \in \mathcal{R}\left(A^{T}\right)$, and $\mathcal{R}\left(R^{\omega}\right) \subset \mathcal{R}\left(A^{T}\right)$.

(ii) Following the reasoning from Theorem 2, the previous part of this result and Lemma 2 imply that the matrix $\Phi^{\alpha}$ satisfies the properties (29) (30).

We will use the following results from [6] and [4] to prove that the assumptions (28) and (31) are true for $\tilde{Q}^{\omega}$ and $\tilde{\Phi}^{\omega}$, respectively.

Lemma 5. [6] The matrices $\tilde{Q}^{\omega}$ and $\tilde{\Phi}^{\alpha}$ are normal matrices.

Theorem 4. [4, Theorem 9] $0<\omega<\frac{2}{\rho(E)}$ if and only if $\rho\left(\tilde{Q}^{\omega}\right)<1$, where $E=\frac{1}{\omega} R^{\omega} A$.

Corollary 1. [6] If $0<\omega<\frac{2}{\rho(E)}$ and $0<\alpha<\frac{2}{\rho(D)}$, where $D=\frac{1}{\alpha}\left(I-\Phi^{\omega}\right)$, then $\tilde{Q}^{\omega}$ and $\tilde{\Phi}^{\alpha}$ satisfy the assumptions (28) and (31), respectively.

Proof. The results in Theorem 4 are also true for $\tilde{Q}^{\omega}$ and $D$. From Lemma 5 we have that $\tilde{Q}^{\omega}$ and $\tilde{\Phi}^{\alpha}$ are normal matrices, which implies that $\left\|\tilde{Q}^{\omega}\right\|=\rho\left(\tilde{Q}^{\omega}\right)$ and $\left\|\tilde{\Phi}^{\alpha}\right\|=\rho\left(\tilde{\Phi}^{\alpha}\right)$. Consequently, Theorem 4 yields that $\left\|\tilde{Q}^{\omega}\right\|<1$ and $\left\|\tilde{\Phi}^{\alpha}\right\|<1$.

From Theorem 3 and Corollary 1 it results that the Extended Block Jacobi with Relaxation Parameters algorithm is a particular case of the EGEN method. 
WEAKER ASSUMPTIONS FOR CONVERGENCE OF EXTENDED BLOCK

\section{Acknowledgements}

We would like to thank our anonymous referees for their helpful suggestions which much improved the first versions of the manuscript.

\section{References}

[1] Ben-Israel, A. and Greville, T., Generalized Inverses: Theory and Applications, Second Edition, Springer-Verlag, New York, 2003.

[2] Björk, A., Numerical Methods for Least Squares Problems, SIAM, Philadelphia, 1996.

[3] Eggermont P.P.B., Herman G.T., Lent A., Iterative Algorithms for Large Partitioned Linear Systems, with Applications to Image Reconstruction, Linear Alg. Appl., 40(1981), 37-67.

[4] Elfving, T., Block-Iterative Methods for Consistent and Inconsistent Linear Equations, Numer. Math., 35, 1-12, 1980.

[5] Nicola, A., Petra, S., Popa, C. and Schnörr, C., A general extending and constraining procedure for linear iterative methods, Intern. J. Comp. Math., 89, 231-253, 2012.

[6] Popa, C., Extensions of block-projections methods with relaxation parameters to inconsistent and rank-deficient least-squares problems, BIT Numerical Analysis 38(1), 151-176, 1998.

[7] Popa, C., Extended and constrained diagonal weighting algorithm with application to inverse problems in image reconstruction, Inverse Probl., 26, doi: 10.1088/0266-5611/26/6/065004, 2010.

[8] Popa, C., Projection algorithms - classical results and developments. Applications to image reconstruction, Lambert Academic Publishing - AV Akademikerverlag GmbH \& Co. KG, Saarbrücken, Germany, 2012.

[9] Tanabe, K., Projection Method for Solving a Singular System of Linear Equations and its Applications, Numer. Math., 17, 203-214, 1971. 
WEAKER ASSUMPTIONS FOR CONVERGENCE OF EXTENDED BLOCK

Doina CARP,

Constanţa Maritime University,

Mircea cel Bătrân 104, 900663 Constanța, România.

Email: doina.carp@gmail.com

Ioana POMPARĂU,

Ovidius University of Constanta,

Mamaia 124, 900527 Constanţa, România.

Email: ipomparau@univ-ovidius.ro

Constantin POPA,

Ovidius University of Constanta,

Mamaia 124, 900527 Constanța, România.

"Gheorghe Mihoc - Caius Iacob" Institute of Statistical Mathematics and

Applied Mathematics,

Calea 13 Septembrie 13, 050711 Bucureşti, România.

Email: cpopa@univ-ovidius.ro 